\title{
Guest Editorial: Semantic Concept Discovery in MM Data
}

\author{
Xiaojun Chang ${ }^{1} \cdot$ Yan Yan $^{2} \cdot$ Liqiang $\mathrm{Nie}^{3}$
}

Published online: 23 January 2018

(C) Springer Science+Business Media, LLC, part of Springer Nature 2018

The explosive increase of multimedia data (i.e., text, image, and video) on the Internet has brought the great challenge of how to effectively index, retrieve and organize these resources. Much research attention has been paid to discover semantic concepts in multimedia data. This special issue aims to collect recent state-of-the-art achievement on semantic concept discovery, especially the work devoted to several new challenges in this field. Another focus of this special issue will be recent advances on semantic event detection, which refers to detect unseen event without any training data. To achieve this goal, a bunch of concept classifiers should be trained beforehand. How to efficiently train these classifiers with millions of videos is still an open problem.

This special issue targets the researchers and practitioners from both academia and industry. In total, 81 submissions have been received. After three rounds of reviews, we finally accepted 40 of them. There are around 40 reviewers involved in the review process.

Xiaojun Chang

cxj273@gmail.com

Yan Yan

tom.yan.555@gmail.com

Liqiang Nie

nieliqiang@gmail.com

1 Carnegie Mellon University, Pittsburgh, PA, USA

2 University of Michigan, Ann Arbor, MI, USA

3 Shandong University, Jinan, China 
Xiaojun Chang received the Ph.D degree in computer science from University of Technology Sydney, Australia. He is currently a Post-Doctoral Research Associate in Carnegie Mellon University. His research interests include machine learning and its applications to multimedia analysis and computer vision. He has authored/co-authored over 40 scientific papers in these areas, which are published in the IEEE Transactions journals (T-PAMI/T-NNLS/ $\mathrm{T}-\mathrm{CYB} / \mathrm{T}-\mathrm{KDE}$ ) and top-tier computer vision and machine learning conferences (CVPR/ICML).

Yan Yan received the Ph.D degree in computer science from University of Trento, Italy. He is currently a PostDoctoral Researcher at University of Michigan. His research interests include machine learning and its application to computer vision and multimedia analysis, e.g. multi-view head pose estimation, multi-view action recognition, multimedia retrieval, image annotation, etc. He is the author of referred journals and conferences in IEEE TPAMI, IEEE TIP, IEEE TNNLS, CVIU, CVPR, ICCV, ACM Multimedia, AAAI, IJCAI, etc. Dr. Yan is the recipient of Best Student Paper Award in ICPR 2014 and Best Paper Award in ACM Multimedia 2015. He was a Visiting Scholar with Carnegie Mellon University in 2013 and Visiting Postdoc Researcher in ADSCUIUC Singapore. He has served as PC member for ACM Multimedia 2013-2015 and reviewer for referred journals and conferences.

Liqiang Nie received the B.E. degree from Xian Jiaotong University, Xian, China, in 2009, and the Ph.D. degree from the National University of Singapore, Singapore, in 2013, both in computer science. He is currently a professor with the School of Computer Science and Technology, Shandong University. He was a Research Fellow with the School of Computing, National University of Singapore. Various parts of his work have been published in top forums, including ACM SIGIR, ACM SIGMM, TOIS, IIST, and IEEE Transactions on Multimedia. His current research interests include information retrieval and healthcare analytics. 